\title{
Angular momentum and overshooting: two as yet unsolved problems in stellar mixing
}

\author{
V. M. Canuto ${ }^{1,2} \dagger$ and Y. Cheng ${ }^{1,3}$ \\ ${ }^{1}$ NASA, Goddard Institute for Space Studies, New York, NY 10025, USA \\ email: vcanuto@giss.nasa.gov, ycheng@giss.nasa.gov \\ ${ }^{2}$ Dept. of Appl. Phys. and Appl. Math., Columbia University, New York, NY 10027, USA \\ ${ }^{3}$ Ctr. Clim. Sys. Res., Columbia University, New York, NY 10025, USA
}

\begin{abstract}
Helioseismological data have given us two interesting results: the differential-touniform solar rotation curve and the extent of the overshooting region (OV). As of today, no model (including numerical simulations) has been able to reproduce these findings. Here, we first present a new model for the angular momentum. It contains new terms representing vorticity and buoyancy that were left out in all previous formulations without a clear justification. It is shown that they extract angular momentum from the stellar core, a welcome feature since the standard angular momentum equation leads to a rotation curve that is considerably higher than what is observed. As for the overshooting extent, all models yield values that are an order of magnitude larger than the helio data of $0.07 H_{p}$. We propose a criterion whose main ingredient is a new flux conservation law that includes new terms, one of which increases the dissipation in the radiative zone and thus lowers the OV extent, a tendency in the desired direction. Since we have not coupled the new models to a solar structure-evolution code, we cannot at this stage carry out a comparison with the helio data. The purpose is to exhibit the fact that in both cases the missing ingredients are of such nature as to improve the previous model predictions. A proper quantification remains to be done.
\end{abstract}

Keywords. Stars: abundances, convection, turbulence

\section{Introduction}

Since mixing in stars is a complex interplay of processes as diverse as unstable stratification, stable stratification, differential rotation, gravity waves, double-diffusion, etc., the formalism employed to model mixing should be sufficiently general to account for such a large variety of processes. And yet, the literature shows that this is not the case, the two methodologies being employed being: a) large scale numerical simulations and b) heuristic models. In the first case (e.g., Brummell et al., 2002), the values of several parameters, such as the Prandtl number, are widely different from those in stars. The authors of those studies have however stated that their primary goal was the elucidation of the intertwined physical processes and not to provide stellar studies with tools to model the processes of interest. The consequence is that mixing processes are still modeled using heuristic arguments that have severe limitations, as we discuss in section 5. Our assessment is that heuristic models should be abandoned for lack of physical completeness. As a substitute we suggest, work out and assess models of at most algebraic complexity that avoid the guessing work that heuristic models always entail. It is instructive to point out that an analogous situation existed in geophysics, specifically in modeling atmospheric and oceanic mixing. More than 25 years ago, it was decided to forgo the heuristic approach in favor of a more predictive and flexible tool known as RSM

$\dagger$ Present address: NASA-GISS, 2880 Broadway, New York, NY 10025, USA. 
(Reynolds Stress Methodology) which is now commonly used. For reasons unclear to the present authors, stellar mixing studies are lagging behind geophysical studies and this paper will thus discuss a new model as well as the limitations of the heuristic models. The RSM is a set of equations for the turbulent correlations of the velocity and temperature fields that follow from the Navier-Stokes equations and the temperature equations. The RMS'main features can be summarized as follows: mathematical structure, the relevant equations are a set of linear algebraic equations and thus pose no particular numerical problems; flexibility, which is one of the key advantages, means that adding new processes such as rotation, vorticity, double-diffusion, etc, does not require guessing work since the RSM has a well defined set of procedural rules; assessment, the results of the RSM can be assessed before being used in a stellar context, an important feature that none of the heuristic model satisfies, raising the justified doubt that these models were constructed and tailored to a specific astrophysical setting, a feature that limits their predictive power. Since the RSM was discussed in detail elsewhere, we refer to that work for more details (Canuto, 2008).

\section{The angular momentum problem}

The angular momentum equation is given by $(\Gamma=\sin \theta)$ :

$$
\frac{\partial}{\partial t}\left(r^{2} \Omega\right)=-\Gamma^{-1} r^{-2} \frac{\partial}{\partial r}\left(r^{3} \tau_{r \phi}\right)-\Gamma^{-3} \frac{\partial}{\partial \theta}\left(\Gamma^{2} \tau_{\theta \phi}\right)+\ldots
$$

where $\tau_{i j}=\bar{u}_{i} u_{j}$ are the Reynolds stresses. If one assumes that $\tau_{r \phi}$ is governed only by shear $S_{i j}$, one has:

$$
\tau_{r \phi}=-2 K_{m} S_{r \phi}, \quad S_{r \phi}=\frac{1}{2} r \Gamma \frac{\partial \Omega}{\partial r}, \quad 2 S_{i j}=\bar{u}_{i, j}+\bar{u}_{j, i}
$$

where $K_{m}$ is a momentum diffusivity, (2.1) then becomes (Talon and Zahn, 1998; Talon and Charbonnel, 2003; Palacios et al., 2003, 2006):

$$
\frac{\partial}{\partial t}\left(r^{2} \Omega\right)=r^{-2} \frac{\partial}{\partial r}\left(r^{4} K_{m} \frac{\partial \Omega}{\partial r}\right)+\ldots
$$

Thompson et al. (2003) have written that (2.3) predicts rotation of the solar interior at a rate several times higher than the surface rate in stark disagreement with helio data of nearly uniform rotation. The first obvious conclusion is that since shear alone does not fully represent the mean flow, one must also include vorticity:

$$
V_{i j}=\frac{1}{2}\left(\bar{u}_{i, j}-\bar{u}_{j, i}\right), \quad V_{r \phi}=-\frac{1}{2} r^{-1} \Gamma \frac{\partial}{\partial r}\left(r^{2} \Omega\right)
$$

which leads to a real "diffuse nature" of the angular momentum. Furthermore, it seems natural that if one wants to explain the different behavior of the solar rotation curve in the convective and radiative zones, one must have an "ingredient" capable of differentiating between the two regimes. The obvious candidate is the buoyancy flux that is positive in the first case and negative in the second. On the basis of these qualitative considerations, one concludes that in addition to shear and vorticity, the Reynolds stresses must also depend on buoyancy and thus $\tau_{i j}(S, V, B)$. Finally, one must account for possible radiative losses and thus the formalism must include a Peclet number. We conclude that the final form of the Reynolds stresses must be:

$$
\tau_{i j}(S, V, B, P e)
$$




\section{Reynolds stresses and heat fluxes}

In the presence of shear, vorticity, buoyancy and radiative losses, the general form of the traceless Reynolds stress tensor $b_{i j}=\tau_{i j}-\delta_{i j} 2 K / 3$ is (Canuto and Minotti, 2001):

$$
A \tau^{-1} b_{i j}=-\frac{8 K}{15} S_{i j}-\frac{1}{2} Z_{i j}+\frac{1}{2} B_{i j}
$$

where

$$
Z_{i j}=b_{i k} V_{j k}+b_{j k} V_{i k}, \quad B_{i j}=\alpha\left(g_{i} J_{j}+g_{j} J_{i}\right)-\frac{2}{3} \delta_{i j} \alpha g_{k} J_{k}
$$

Here, $A=5, \alpha=-\rho_{0}^{-1} \partial \bar{\rho} / \partial T$ is the volume expansion coefficient and $J_{i}=\overline{u_{i} \theta}$ is the heat flux for which the RSM provides the following equations (Canuto and Minotti, 2001):

$$
\begin{gathered}
\tau^{-1} A_{i j} J_{j}=-\tau_{i j} \frac{\partial T}{\partial x_{j}}, \quad \tau=2 K \epsilon^{-1} \\
A_{i j}=\lambda_{5} \delta_{i j}+\lambda_{6} \tau S_{i j}+\lambda_{7} \tau V_{i j}+\lambda_{8} \tau^{2} \alpha g_{i} \frac{\partial T}{\partial x_{j}}+2 \epsilon_{i p j} \Omega_{p}^{0}
\end{gathered}
$$

with $\lambda_{6,7,8}=0.786,0.643,0.547$. Canuto and Dubovikov (1998) and Canuto (2008) showed that the Peclet number dependence enters via the two remaining variables:

$$
\begin{gathered}
\lambda_{5}^{-1}=a P e(1+b P e)^{-1}(1+R i)^{-1}, \quad \lambda_{8}=c P e(1+d P e)^{-1} \\
a=\left(4 \pi^{2}\right)^{-1}, \quad b=5 a\left(1+\sigma_{t}^{-1}\right), \quad c=\frac{8}{3}\left(7 \pi^{2}\right)^{-1}, \quad d=4\left(7 \pi^{2}\right)^{-1} \sigma_{t}^{-1}
\end{gathered}
$$

where $\sigma_{t}=0.72$ and $R i$ is the Richardson number which is present only in the stable stratification case.

\section{New results of the RSM model}

We have numerically solved the set of linear algebraic Eqs.(3) and in figure 1 we present the heat diffusivity as a function of $R i$ and for different values of $P e$; in figure 2 we plot the momentum diffusivity while in Fig.3 we plot the turbulent Prandtl number $\sigma_{t}(R i, P e) \equiv K_{m} / K_{h}$, which is the ratio between momentun and heat diffusivities. As one can see, the ratio in not constant but increases with $R i$. For large $P e$, we have superimposed a variety of LES, DNS, lab and direct measurements (Canuto et al., 2008) that the model reproduces quite well.

An example of the results that are obtained from our method is given in figure 1.

\section{Previous heuristic mixing model}

Here, we compare the results of Figs.1-3 with those of the heuristic relations used by various authors (Mathis et al., 2004; Palacios et al., 2003, 2006; Charbonnel and Talon, 2005, 2007; Maeder and Meynet, 2001):

$$
P e>>1: \quad \frac{K_{m, h}}{\chi}=2 \frac{R i(c r)}{R i}=\frac{1}{3 R i}
$$

where $\chi\left(\mathrm{cm}^{2} \mathrm{~s}^{-1}\right)$ is the radiative diffusivity. (5.1) is consistent with Fig. 1 if:

$$
P e \approx 10^{2}
$$




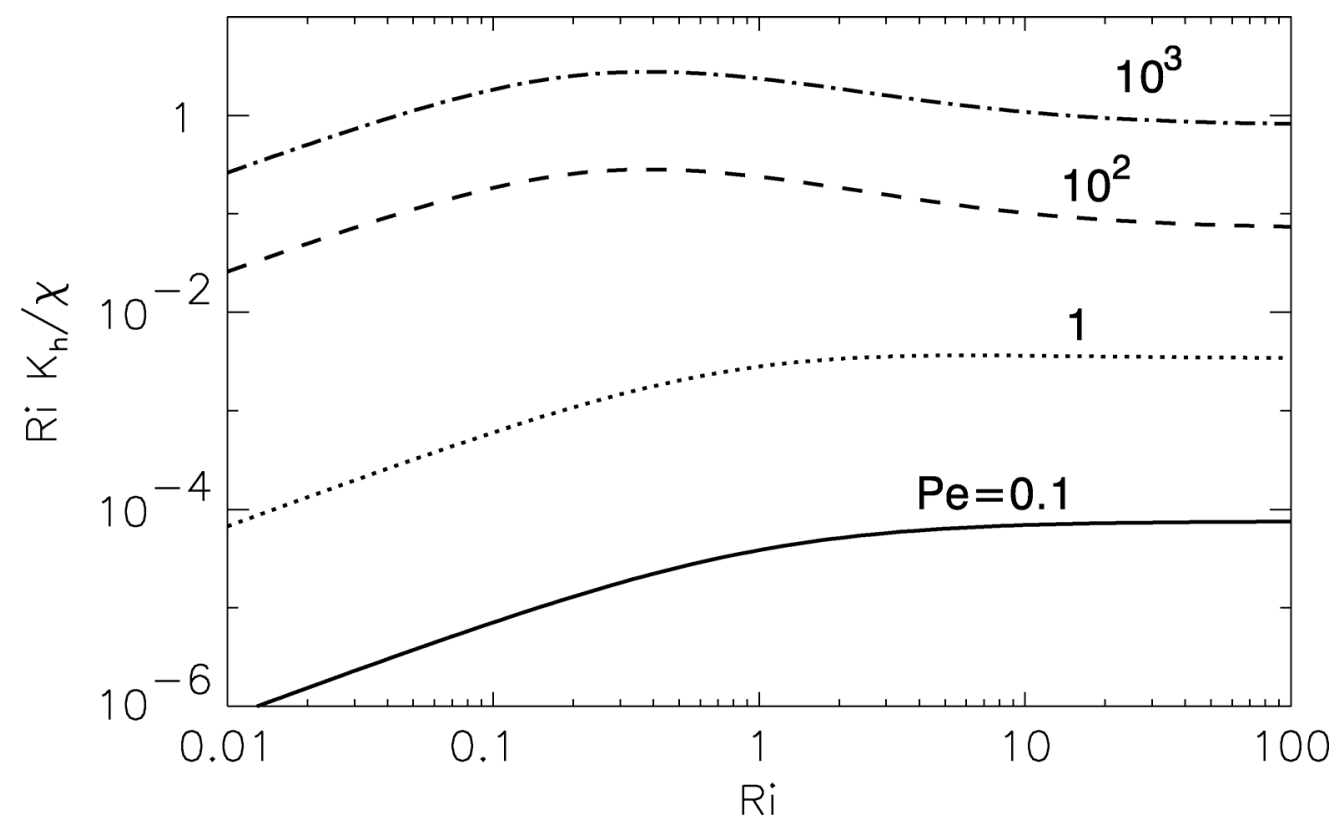

Figure 1. The heat diffusivity $K_{h}$ in units of the radiative diffusivity vs. the Richardson number $R i$ for different values of the Peclet number. As expected, the stronger the effect of stable stratification, the larger is the value of $R i$ and the smaller is the resulting diffusivity. We plot $K_{h}$ multiplied by $R i$ in order to allow a direct comparison with the heuristic model (5.1). See the text for details.

As for the momentum diffusivity in Fig. 2, (5.1) is not satisfied. The only possibility is to impose (5.1) but that in turn implies a unique value of $R i$ equal to:

$$
R i \approx 0.1
$$

This means that the heuristic model is valid for only one combination of $P e, R i$ given by (5.2) and (5.3) which is quite unusual for any model. Finally, (5.1) implies a turbulent Prandtl number of unity whereas Fig. 3 shows that it is a rather strong function of $R i$ unless one limits the validity of the model to $R i<<1$.

\section{New angular momentum equation}

Using a method of symbolic algebra, we have solved Eqs. (3) without meridional currents. Introducing three dimensionless variables $x, X$ and $Z$ to characterize stratification, shear and vorticity in units of the dynamical time scale $\tau=2 K / \epsilon$ :

$$
x \equiv(\tau N)^{2}, \quad X \equiv \tau S_{r \phi}, \quad Z \equiv \tau V_{r \phi}
$$

the explicit form of the Reynolds stress that enters (2.1) turns out to be:

$$
\tau_{r \phi}=-2 K_{m}^{(1)} S_{r \phi}-2 x K_{m}^{(2)} V_{r \phi}
$$

which yields the new angular momentum equation to:

$$
r^{2} \frac{\partial}{\partial t}\left(r^{2} \Omega\right)=\frac{\partial}{\partial r}\left(K_{m}^{(1)} r^{4} \frac{\partial \Omega}{\partial r}\right)+\frac{\partial}{\partial r}\left(x K_{m}^{(2)} r^{2} \frac{\partial r^{2} \Omega}{\partial r}\right)
$$

Several considerations are in order:1) the presence of vorticity has now introduced a new term which has the character of a true diffusion of angular momentum whereas the 


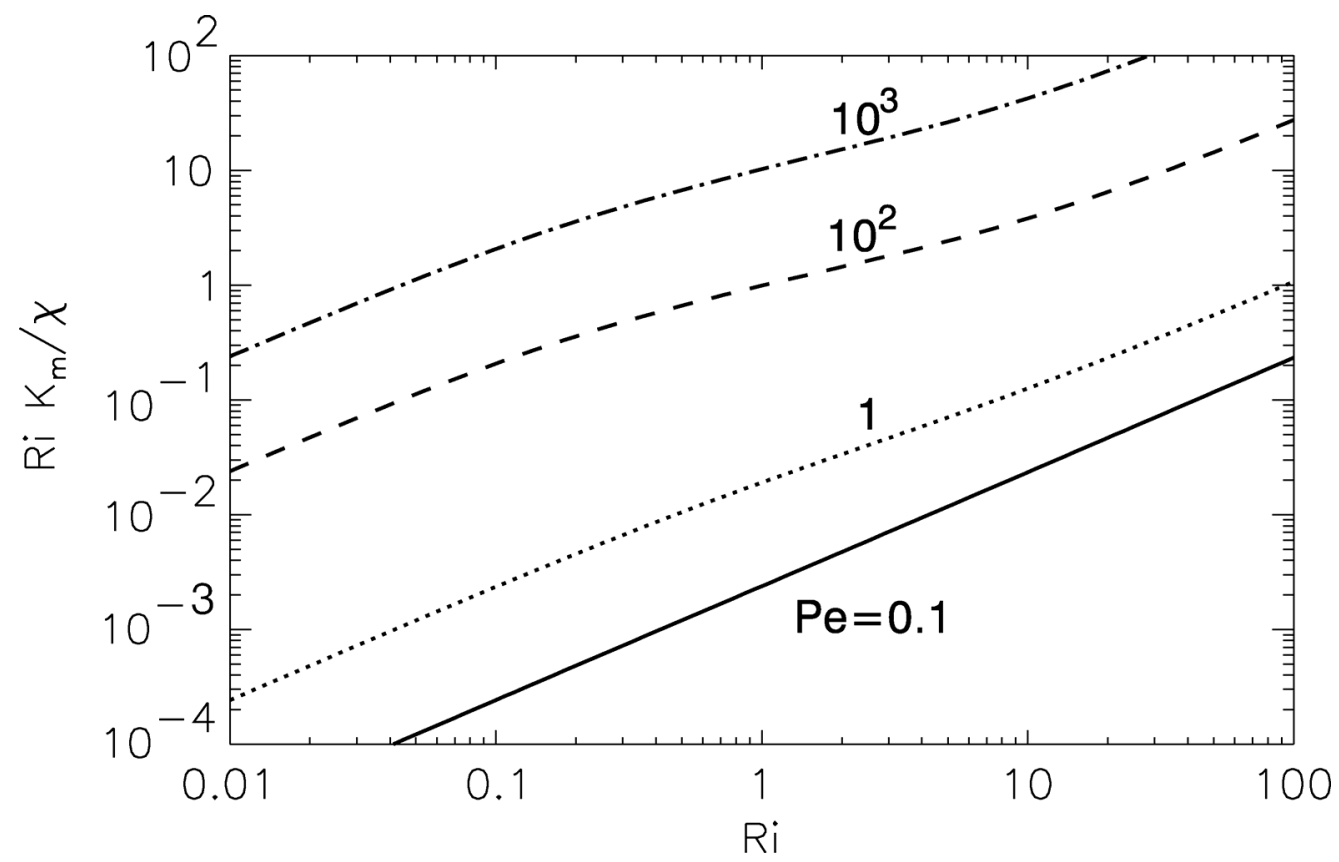

Figure 2. Same as in figure 1 but for the momentum diffusivity $K_{m}$.

first traditional term, in spite of being usually called "diffusion", is not, 2) the new term entails vorticity multiplied by buoyancy represented by the variable $x$ which is negative in the convective zone and positive in the radiative, stably stratified, zone, 3 ) in the latter zone, where we can take $\Omega=$ constant as from the helio data, only the second term in (6.3) survives and since $x>0$, this implies an outward transport of angular momentum from the radiative interior, an "extraction process" that in principle drives it toward a state of uniform rotation, 4) since in the radiative zone turbulence is much weaker than in the $\mathrm{CZ}$, the eddies life time is correspondingly longer and the variable $x$ is an increasing function of $R i$ making its largest contribution to the second term in (6.3), $5)$ the two momentum diffusivities are not the same since they themselves depend on $x$, $X, Z$ but for the purposes of this paper their expressions are not relevant (they can be provided by request to the authors). 6) even if one assumes that the two diffusivities in (6.3) are the same and of the form (5.1), the first term in (6.3) decreases like $R i^{-1}$ while the combination $x K_{m}$ decreases with $R i$ with a lower power.

In summary, the inclusion of both buoyancy and vorticity leads to a new angular momentum equation which may provide a better model for the helio data since it contains a mechanism to extract angular momentum from the stellar core that is absent in the commonly used formula (2.3).

\section{New equation for the OV extent}

As for the OV extent, numerical simulations (Brummel et al., 2002) yield a value of about $0.5 H_{p}$ which is an order of magnitude larger than the helio data of $0.07 H_{p}$. To reconcile model results with the data, we suggest a new criteria for the OV extent. Consider the equation for the turbulent kinetic energy $K\left(D / D t \equiv \partial / \partial t+\bar{u}_{i} \partial / \partial x_{i}\right)$ :

$$
\frac{D K}{D t}+\frac{\partial F_{i}^{k e}}{\partial x_{i}}=P_{b}+P_{m}-\epsilon
$$




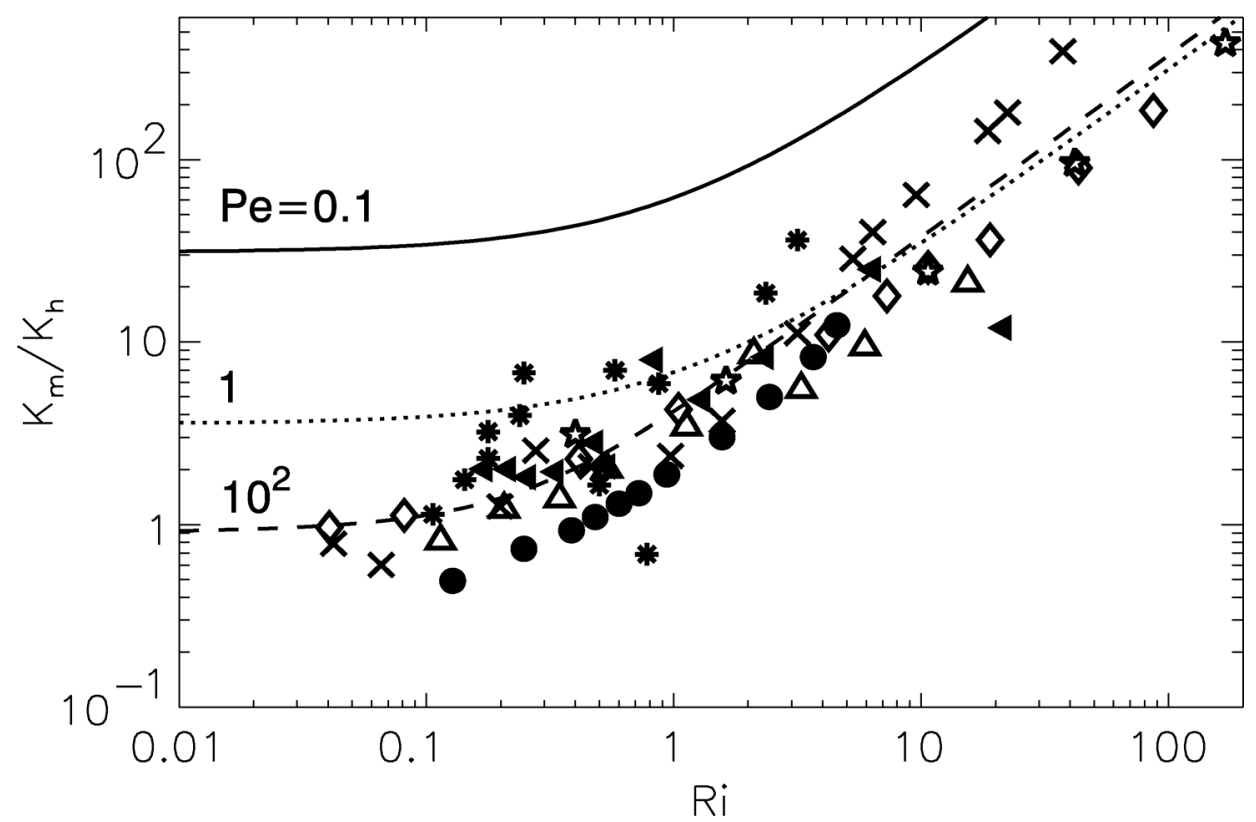

Figure 3. Turbulent Prandtl number $\sigma_{t}=K_{m} / K_{h}$ vs. $R i$ for different $P e$. The data corresponding to the $P e>1$ case, are as follows: meteorological observations (Kondo et al., 1978, slanting black triangles; Bertin et al., 1997, snow-flakes), lab experiments (Strang and Fernando, 2001, black circles; Rehmann and Koseff, 2004, slanting crosses; Ohya, 2001, diamonds), LES (Zilitinkevich et al., 2007, 2008, triangles), DNS (Stretch et al., 2001, five-pointed stars).

where $F_{i}^{k e}$ is the flux of $K$ and $P_{b, m}=\left(\alpha g_{i} J_{i},-\tau_{i j} S_{i j}\right)$ are the productions of buoyancy and shear. Next, consider the flux conservation law (Canuto, 1997):

$$
\begin{gathered}
F_{i}^{r a d}+F_{i}^{c o n v}+F_{i}^{k e}+\bar{u}_{j}\left(\mathbf{E} \delta_{i j}+\tau_{i j}\right)=\text { constant }=C \\
E=c_{p} T+K+\bar{K}+G, \quad \bar{K}=\frac{1}{2} \overline{\mathbf{u}}^{2}, \quad g_{i} \bar{u}_{i}=\frac{D G}{D t}
\end{gathered}
$$

where $F_{i}^{\text {conv }}=c_{p} J_{i}$. While the "traditional" flux conservation law used in stellar models contains only the first two terms in (7.2), one must also account for the flux of $K$ represented by the third term; however, in the presence of mean currents, one has new terms, the first of which represents the flux $\bar{u}_{i} \mathbf{E}$, where $\mathbf{E}$ is the sum of enthalpy $c_{p} T$, turbulent kinetic energy $K$, mean field kinetic energy $\bar{K}$ and gravitational energy $G$ while the other term is the flux of the Reynolds stresses. Eliminating the heat flux between (7.1) and 7.2 ), yields in the stationary case the equation for the flux of $\mathrm{K}$ :

$$
\begin{gathered}
\frac{\partial F_{i}^{k e}}{\partial x_{i}}+\frac{\alpha}{c_{p}} g_{i} F_{i}^{k e}=\Phi_{o l d}+\Phi_{n e w} \\
\Phi_{\text {old }}=C-\alpha c_{p}^{-1} g_{i} F_{i}^{r a d}, \quad \Phi_{n e w}=-\tau_{i j} S_{i j}-\alpha c_{p}^{-1} g_{i}\left(\tau_{i j} \bar{u}_{j}+\mathbf{E} \bar{u}_{i}\right)-\epsilon
\end{gathered}
$$

After some algebra, one obtains that:

$$
\Phi_{n e w}=-\left(1+T_{2} \frac{r}{H_{p}}\right) \epsilon+\left(T_{0}+T_{1}\right) \epsilon-H_{p}^{-1}\left(\mathbf{E}+\overline{u_{r}^{2}}\right) \bar{u}_{r}
$$

The key result is that the first term shows that the dissipation increases with depth thus reducing the extent of the OV compared to the standard criterion without $\Phi_{n e w}$. This is 
predicated on the fact that:

$$
T_{2} \sim(\tau N)^{2}(\tau \Omega)^{2}
$$

is positive in the radiative zone since $N^{2}>0$. At the same time, the eddies life time is the largest since turbulence is weak and thus $(\tau N)^{2}>1$.

In conclusion, the new term in the OV equation (7.4) contributes a term that reduces the $O V$ extent which is a desired feature since no model has thus far been able to reproduce the helio data of $O V \approx 0.07 H_{p}$.

\section{Conclusions}

The form of the standard angular momentum equation (2.3) yields results that are not in agreement with helio data since the extraction of angular momentum from the radiative zone is too inefficient. We show that (2.3) is based on a very restricted form of the Reynolds stresses. If one uses the full form of the Reynolds stresses that entails shear, vorticity and buoyancy, the combination of the last two ingredients gives rise to a new term that is larger than the canonical one that contains only shear and which leads to extraction of angular momentum from the core, a tendency in the right direction.

As for the OV extent, the key ingredient is the new flux conservation law that entails Reynolds stresses and mean flows. One of the new terms leads to an increased dissipation of the flux of turbulent kinetic energy which in turn entails a smaller OV extent, a welcome feature since thus far all models have yielded an OV extent about an order of magnitude larger than the helio data of $0.07 H_{p}$.

An interesting aspect of the new models is the relative simplicity of the equations determining Reynolds stresses and heat fluxes since they are given by linear algebraic equations. This is especially relevant if one considers the amount of information they contain: stable stratification, unstable stratification, rigid rotation, shear, and radiative losses (Peclet number). Having established the qualitative behavior of the two models, what is needed next is a specific computation in conjunction with a stellar code.

\section{Acknowledgements}

One of the authors (VMC) would like to thank to Dr. F. Kupka for very helpful suggestions and information on a variety of topics discussed in this paper.

\section{References}

Bertin, F., Barat, J., \& Wilson, R. 1997, Radio Science 32, 791

Brummell, N. H., Clune, T. L., \& Toomre, J. 2002, ApJ 570, 825

Brun, A. S. \& Toomre, J. 2002, ApJ 570, 865

Canuto, V. M. 1997, ApJ 482, 827

Canuto, V. M., Minotti, F., \& Shilling, O. 1994, ApJ 425, 303

Canuto, V. M. \& Dubovikov, M. S. 1998, ApJ 493, 834

Canuto, V. M. \& Minotti F. 2001, Mon. Not R. Astron. Soc. 328, 829

Canuto, V. M., Cheng, Y., Howard, A. M., \& Esau, I. N. 2008, J. Atmos. Sci., in press

Canuto, V. M. 2008, in: W. Hillebrandt \& F. Kupka (eds.), Interdisciplinary Aspects of Turbulence, Lecture Notes in Physics (Berlin: Springer)

Charbonnel, C. \& Talon, S. 2005, Science 309, 2189

Charbonnel, C. \& Talon, S. 2007, Science 318, 922

Kondo, J., Kanechika, O., \& Yasuda, N. 1978, J. Atmos. Sci. 35, 1012

Maeder, A. \& Meynet, G. 2001, A\&BA 373, 555

Mathis, S., Palacios, A., \& Zahn, J. P. 2004, A\&A 425, 243

Ohya, Y. 2001, Boundary-Layer Meteorol. 98, 57 
Palacios, A., Talon, S., Charbonnel, C., \& Forestini, M. 2003, A\&A 399, 603

Palacios, A., Charbonnel, C., Talon, S., \& Seiss, L. 2006, A\&A 453, 261

Rehmann, C. R. \& Koseff, J. R. 2004, Dynamics of Atmospheres and Ocean 37, 271

Roxburgh, I. W. 1978, A\&̈A 65, 281

Strang, E. J. \& Fernando, H. J. S. 2001, J. Phys. Ocean. 31, 2026

Stretch, D. D., Rottman, J. W., Nomura, K. K., \& Venayagamoorthy, S. K. 2001, in: B.B. Dally (eds.), Proc. Fourteenth Australasian Fluid Mech. Conf., Adelaide University, South Australia, 612-628

Talon, S. \& Zahn, J. P. 1998, A\&A 329, 315

Talon, S. \& Charbonnel, C. 2003, A $\& A$ 405, 1025

Thompson, M. J., Christensen-Dalsgaard, J., Miesh, M. S., \& Toomre, J. 2003, Annu. Rev. Astron. Astrophys. 41, 599

Zilitinkevich, S. S., Elperin, T., Kleeorin, N., \& Rogachevskii, I. 2007, Boundary Layer Meteorol. 125,167

Zilitinkevich, S. S., Elperin, T., Kleeorin, N., Rogachevskii, I., Esau, I., Mauritsen, T., \& Miles, M. W. 2008, Quart. J. Roy. Meteor. Soc., in press

\section{Discussion}

LANGER: For obtaining angular momentum transport in radiative zones, do you assume turbulence to exist?

CAnuto: I do assume, as does everybody else, that in the radiative zone there is a variety of instabilities which ultimately will give give rise to a turbulent flow.

ZAHN: You mentioned the fact that numerical simulations predict an overshoot which is much too strong. But that is so because such simulations are run with a Peclet number which is too low, owing to the lack of resolution. For a given strength of the convection, the higher thermal diffusion, i.e. the lower the Peclet number, and the deeper is the overshoot, because the buoyancy force is lessened.

CAnuto: One thing is what simulations do and another is what the physics of the problem dictates - since the Peclet's number is directly proportional to the rsm turbulent velocity and since the latter is getting smaller as one approaches the bottom of the $\mathrm{CZ}$, so does the Peclet's number - inside the radiative region, such rsm velocity is even smaller and so is Pe - Thus, small Pe, small rsm and small OV distance go together. 